\title{
An Integrated System for Digital Restoration of Prehistoric Theran Wall Paintings
}

\author{
Nikolaos Karianakis \\ Computer Science Department \\ UCLA \\ Los Angeles, USA \\ nikos.karianakis@gmail.com
}

\author{
Petros Maragos \\ School of Electrical and Computer Engineering \\ NTUA \\ Athens, Greece \\ maragos@cs.ntua.gr
}

\begin{abstract}
We present a computer vision system for robust restoration of prehistoric Theran wall paintings, replacing or just supporting the work of a specialist. In the case of significant information loss on some areas of murals, the local inpainting methods are not sufficient for satisfactory restoration. Our strategy is to detect an area of relevant semantics, geometry and color in another location of the wall paintings, which in turn is stitched into the missing area by applying a seamless image stitching algorithm. An important part of our digital restoration system is the damaged and missing areas detector. It is used in combination with total variation inpainting at first for the missing area extraction and repair, and secondly for the elimination of minor defects on the retrieved part in the non-local inpainting mechanism. We propose a morphological algorithm for rough detection and we improve upon this approach by incorporating edge information. For missing areas with complicated boundaries we enhance the detection by using iterated graph cuts.

Index Terms-prehistoric wall paintings, digital restoration, segmentation, inpainting, image stitching, mathematical morphology, variational methods.
\end{abstract}

\section{EDICS Category: Image/Video Processing Techniques}

\section{INTRODUCTION}

Over the last decade there has been a trend toward computer-aided virtual reconstruction of cultural heritage. Here we describe our contributions ${ }^{1}$ to the digital restoration of the 3600-year-old murals excavated in Akrotiri, which is a prehistoric Aegean settlement in the Greek island of Thera (Santorini).

The Theran wall paintings are famous worldwide for their great artistic and historical value. The task of restoring them is demanding and time-consuming, requiring the great effort of several specially trained conservators. The restoration process comprises, among other things, gathering the fragments from the archaeological site, cleaning each fragment in the lab, and reassembling each composition from its constituent fragments. Our main goal is to virtually restore the reassembled wall paintings by detecting and filling in the missing parts.

\footnotetext{
${ }^{1}$ This work was mainly performed while the first author was at the National Technical University of Athens (NTUA).
}

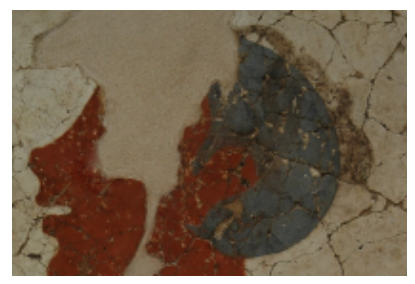

(a) Big missing part

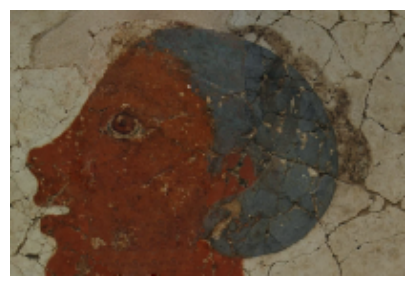

(b) Our restoration result
Fig. 1. Major damage appears in an area of the Theran wall painting Men in Procession, which is restored by retrieving non-local information.

Some previous work on digital painting restoration or pattern detection and fragment synthesis problems in wall paintings include the following. Giakoumis et al. [9] detect the cracks on digitized paintings by using the morphological top-hat transform. Then they remove the thin dark brush strokes, which are misidentified as cracks, by training an MRBF neural network. In [19] and [4] the fragments geometry is examined and then this information is exploited for wall painting reconstruction from their constituent parts via matching algorithms. Papaodysseus et al. in [18] analyze and reconstruct some characteristic patterns in Theran murals, such as spirals, and infer conclusions regarding their painting method. Shin et al. [25] use interactive hardware to trace fragment boundaries and geometric analysis algorithms to study their shapes and contacts, so that they create a statistical model for hierarchical fragment synthesis.

In this paper, we propose an integrated system which restores missing parts of various shapes and sizes that appear in Theran wall paintings. At first, we suggest a mathematical morphology algorithm that also incorporates edge information for detecting missing areas. Then, in case of smaller cracks we apply inpainting based on total variation ( [1], [2], [6], [16], [24]). Otherwise, when the damage is major, we provide a better segmentation by using iterated graph cuts and apply template matching to search for non-local information. The latter is stitched to the gap seamlessly. Unlike other scene completion methods (e.g. [10]), we do not train our model, but we just search automatically everywhere on the wall 
paintings, which is efficient as the database is limited due to the nature of the problem. Fig. 1 shows a result of our method.

\section{Crack Detection \& InPainting}

Our method for crack detection is based on morphological feature detection [15]. In the case of cracks and small missing areas, finding their boundaries and grouping them is enough for their rough identification. Furthermore, ensuring the coverage of major edges brings more complete and precise recognition. The Canny algorithm [5] is used for edge detection. Then, total variation inpainting [24] follows, so that we fill in the missing areas smoothly.

As the missing area boundary presents high intensity variation in at least one direction, thresholding the discrete image derivative along the two main axes ensures a rough boundary detection. Rigorously speaking, let's consider the image $I(i, j), 1 \leq i \leq N_{1}, 1 \leq j \leq N_{2}$ of the wall painting area to be restored. We consider as cracks the set of pixels

$$
C=\left\{(i, j): \max \left(\left|\partial_{x} I(i, j)\right|,\left|\partial_{y} I(i, j)\right|\right)>\theta\right\}
$$

at which the magnitude of the image derivatives $\left(\partial_{x} I, \partial_{y} I\right)$ along at least one of the two main axes $x, y$ has value above the threshold

$$
\theta=k \cdot\left(\sum_{i} \sum_{j} \sqrt{\left(\partial_{x} I(i, j)\right)^{2}+\left(\partial_{y} I(i, j)\right)^{2}}\right) /\left(N_{1} N_{2}\right) .
$$

The remaining pixels are considered as genuine at this first stage. To group the neighboring pixels belonging to cracks, we apply morphological closing using a disk with radius $\ell$ pixels:

$$
R=C \bullet \ell B=(C \oplus \ell B) \ominus \ell B
$$

where $B$ is the unit disk. In that way, greater consistency is achieved in the missing areas. At this point the region based binary mask of cracks $R$ has been extracted.

Based on the fact that the crack boundaries are often edges, the Canny edge detection method is used for enhanced crack detection. The binary mask $E$ of edge pixels is dilated using a disk $m B$ with an $m$-pixel radius, so that neighboring edges are grouped. Then erosion with the unit disk $B$ is applied iteratively at the logical disjunction between the binary images of dilated edges $E \oplus m B$ and region based mask $R$ :

$$
\begin{gathered}
\qquad M=(E \oplus m B) \bigcup R \\
\text { while }(|M \bigcap E| \geq(1-e)|E|) \text { do } M=M \ominus B
\end{gathered}
$$

where $|\cdot|$ denotes area. The loop stops before more than $e \%$ of edge information is lost. Thus, we drop part of the redundant information, receive a smoother version of the mask and keep inside the recognized-as-cracks region the pixels that with high probability are part of the missing areas. The whole procedure combines both region- and boundary-based processing and is briefly described by Alg. 1. Two examples

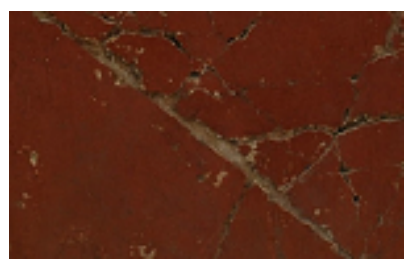

(a) Damaged area

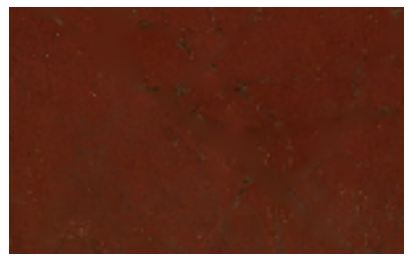

(d) Restored area

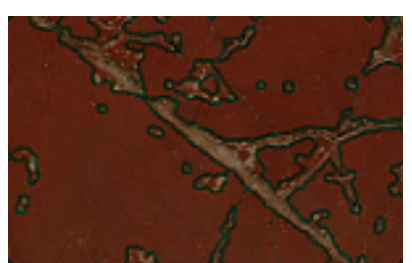

(b) Crack detection

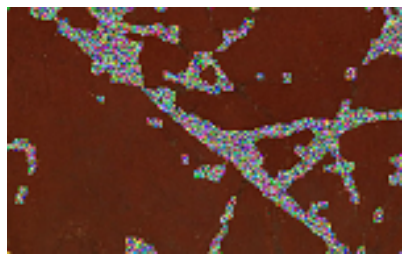

(c) Initialization of inpainting
Fig. 2. The first example of crack detection using Alg. 1. Total variation inpainting is applied in turn for the cracks restoration.

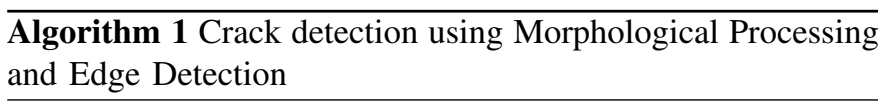

Exploiting gradient information

- We find the areas $C$ where our image presents high relative intensity variation along some of the main axes $\mathrm{x}$ and $\mathrm{y}$.

- We apply morphological closing with disk $\ell B$, where $B$ is the unit disk, so that we find the region based mask $R$. Exploiting edge information

- In the core of the boundary based part, the Canny method is used for edge detection.

- Dilation with a disk $m B$ is applied on the binary image of edges $E$.

- We calculate the logical disjunction $M$ of the binary images of dilated edges $E \oplus m B$ and region based mask $R$.

- Erosion with the unit disk $B$ is applied on the mask $M$, iteratively. The algorithm stops just before we lose more than $e \%$ of edge information.

of crack detection in the wall painting Men in Procession are presented in Figs. 2 and 4.

As quality criteria for the evaluation of the crack detector we use the precision, which is the fraction of retrieved instances that are relevant, and the recall, which is the fraction of relevant instances that are retrieved. By incorporating the edge information, we achieve both higher recall and precision. Some statistics about how the error margin $e$ affects the crack detection quality for the example of Fig. 2 are provided in Table I. To this end, we manually made a ground truth mask of cracks. A higher allowable loss of edge information $e$ leads to higher precision, but lower recall. That comes intuitively because the mask of cracks becomes "tighter" (higher precision), but some edge pixels are "lost" at the same time (lower recall). In our case the most important factor is the recall, which exceeds $90 \%$ when $e=1 \%$. High 
TABLE I

CRACK DETECTOR'S QUALITY STATISTICS FOR THE EXAMPLE OF FIG. 2

\begin{tabular}{|c|c|c|}
\hline e $(\%)$ & recall $(\%)$ & precision $(\%)$ \\
\hline \hline 1 & 93.2 & 49.7 \\
\hline 30 & 85.7 & 59.4 \\
\hline 70 & 53.0 & 70.4 \\
\hline
\end{tabular}

recall means that the majority of crack pixels are recognized as such, which is necessary for "proper" inpainting. During restoration the crack pixels that are misclassified as genuine "transmit" their incorrect information inside the crack area.

Therefore, having found the missing areas, we apply total variation inpainting for their restoration. The model and the proposed numerical scheme in [24] were used. Let $D$ be an inpainting domain with boundary $\Gamma$ and $E$ any closed domain in the complement $D^{c}$ that encloses $D$ (Fig. 3). The variational inpainting model finds the function $I$ on the domain $E \cup D$ that minimizes the functional

$$
\int_{E \cup D}|\nabla I| \mathrm{d} x \mathrm{~d} y
$$

under a noise constraint on $E$

$$
\frac{1}{\operatorname{Area}(E)} \int_{E}\left(I-I_{0}\right)^{2} \mathrm{~d} x \mathrm{~d} y=\sigma^{2} .
$$

where $I_{0}$ is the noisy image and $\sigma$ is the standard deviation of white noise. Therefore, the unconstrained model is

$$
J_{\lambda}[I]=\int_{E \cup D}|\nabla I| \mathrm{d} x \mathrm{~d} y+\frac{\lambda}{2} \int_{E}\left(I-I_{0}\right)^{2} \mathrm{~d} x \mathrm{~d} y
$$

where $\lambda$ is the Lagrange multiplier for the constrained TV inpainting problem (5), (6). Thus, along with the inpainting, denoising takes place in the considered-as-genuine area.

The implemented numerical scheme to solve (7) is iterative. We used the following convergence criterion:

$$
\sum_{i} \sum_{j}\left\|I_{n}(i, j)-I_{n-1}(i, j)\right\|<c \cdot \sum_{i} \sum_{j} I_{n}(i, j)
$$

where $n=\frac{\text { iterations }}{100}$ and $c=0.005$. Some results of applying TV inpainting inside the recognized-as-cracks areas by the Alg. 1 are depicted in Figs. 2 and 4. The other constants have

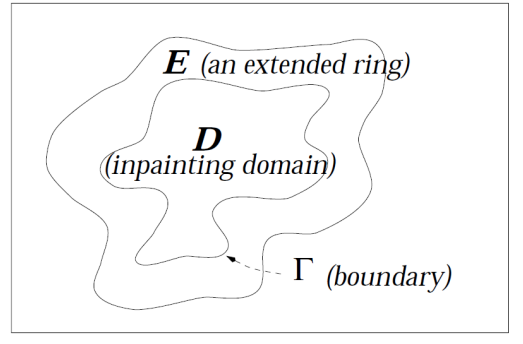

Fig. 3. The TV inpainting model guesses $\left.u\right|_{D}$ by minimizing the TV norm on $E \cup D$ and considering the noise constraint on $E$. [24]

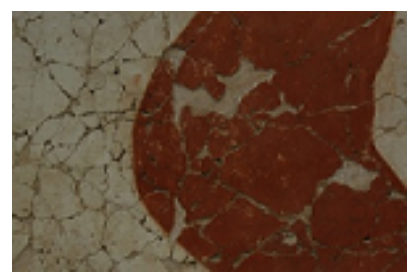

(a) Area with major cracks

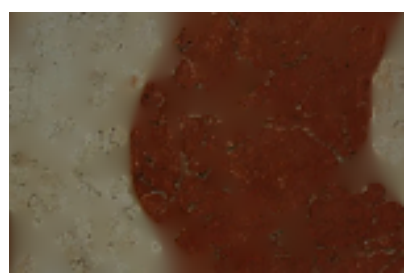

(d) Restored area



(b) Missing areas detection

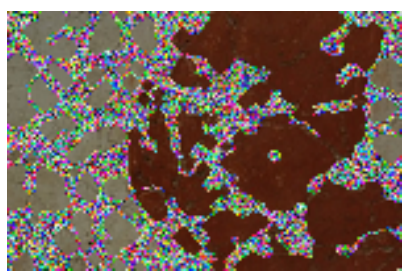

(c) Initialization of inpainting
Fig. 4. The second example of crack detection using Alg. 1 and subsequently TV inpainting for the missing areas restoration.

the following values in each case: $k=1.5, l=4, m=3$, $e=1 \%$ for the example of Fig. 2 and $k=3.6, l=5, m=2$, $e=1 \%$ for the example of Fig. 4. The algorithm converged in 1000 and 600 iterations, respectively.

\section{AN INTEGRATED RESTORATION SYSTEM}

In this section our integrated system for automatic restoration is presented. It provides robust inpainting even in some cases of major damage by retrieving relevant information from other areas in the wall paintings. The system is outlined in Fig. 6.

At first, we define the area to be restored. Then a method for the crack detection is selected depending on the nature of the missing area. In case of small cracks we use Alg. 1 combined with TV inpainting to fill in the gaps smoothly. It is exactly the procedure that has been described in Section II. Although Alg. 1 may give very good results for smaller cracks, it is not equally effective for the detection of larger information gaps. In these cases, we use semi-supervised segmentation algorithms, such as GrabCut [23]. The user roughly defines the background and the foreground and then GrabCut provides the segmentation.

In GrabCut, after the user has defined the background $T_{B}$ and the foreground region $T_{F}$, there is a transient area $T_{U}$ between them, which is unknown:

$$
T=\left\{T_{B}, T_{F}, T_{U}\right\}=\left\{T_{B}, T_{F},\left(T_{B} \cup T_{F}\right)^{c}\right\}
$$

At each pixel in this area a GMM component is assigned, whose parameters are learnt from image data. Then a Gibbs energy with a smoothness term is minimized using $\mathrm{min}$ cuts. GrabCut is a two-step iterative algorithm in which the parameters learning and the energy minimization are 


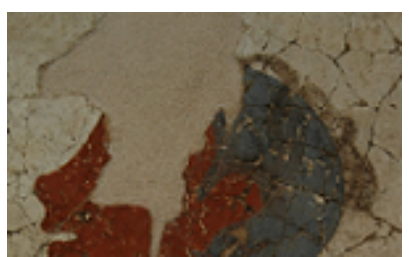

(a) Big missing part

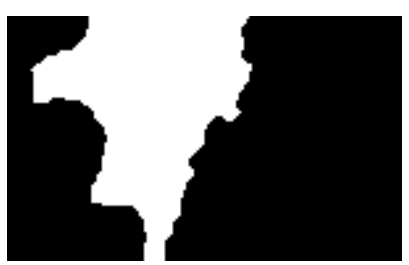

(c) Smoothing
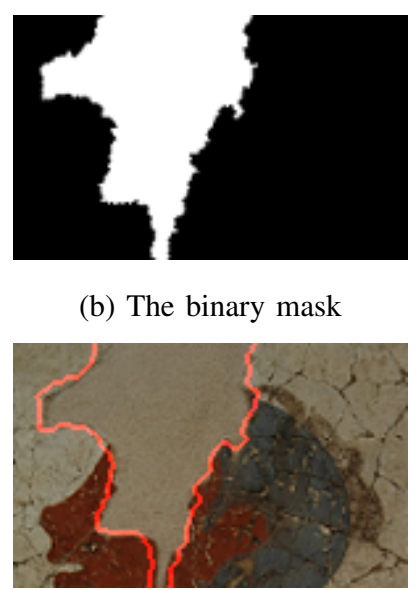

(d) Mask's outline over the image (b) The binary mask

Fig. 5. Missing area detection using GrabCut.

executed alternately. When the algorithm has converged, the pixel values, which are 0 or 1 (background or foreground), correspond to the segmentation. In our case the foreground is considered as the cracks region.

As an alternative approach for our system's crack detection part, Alg. 1 is used for a rough detection $M$ and then we initialize the trimap $T$, so that we define the background, the foreground and the unknown region, respectively, as follows:

$$
T=\left\{T_{B}, T_{F}, T_{U}\right\}=\left\{(M \oplus D)^{c}, M \ominus D,\left(T_{B} \cup T_{F}\right)^{c}\right\}
$$

where $D$ is a disk whose radius is an index of our lack of confidence for the initial segmentation. Then GrabCut provides a quite accurate recognition of missing areas $M^{\prime}$. These steps are described in Alg. 2.

Next, we run a template matching algorithm. Our goal is to detect an area of similar semantics, geometry and color in a different location of the wall paintings in order to cover the big missing area. Thus, the template is the neighborhood of the detected missing area, the search space is the rest surface of all digitally available murals, and the distance is the $L_{1}$ norm. The minimum distance between the template and each possible position in the search space determines the retrieved part, which is the area of the relevant patch that corresponds to the information gap of the genuine patch. Searching takes place in all angles, positions and symmetrically. If

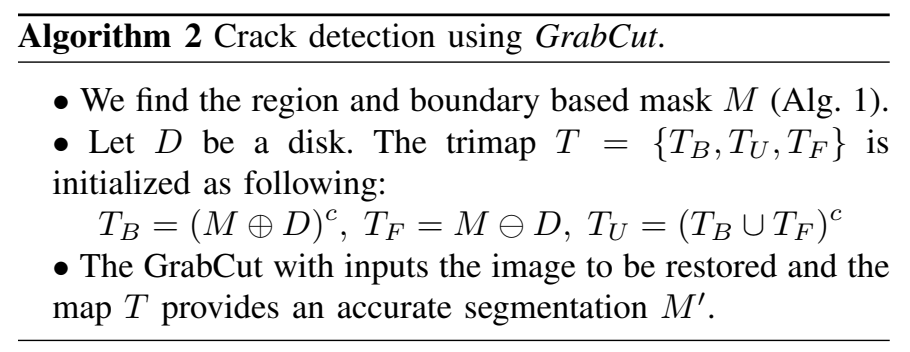

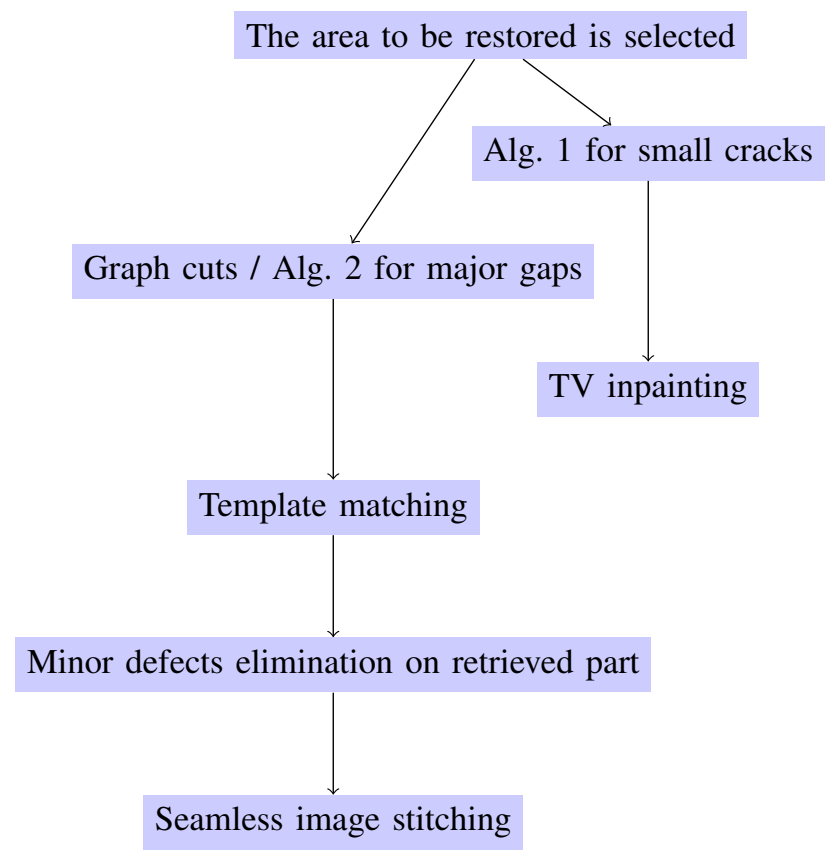

Fig. 6. An integrated system for digital wall painting restoration.

the retrieved part has minor defects, Alg. 1 is used for its restoration. It is considered as appropriate for the restoration, if and only if it misses at most $5 \%$ of its genuine pixels.

Finally, the algorithm by Tao et al. [26] is used for seamless image stitching, where the retrieved part $F$, the area to be restored $B$ and the output $M^{\prime}$ of Alg. 2 are the foreground, the background and the mask, respectively. It is a gradient domain reconstruction method in which we seek the image $I$, so that:

$$
I=\operatorname{argmin}_{f} \int\|\nabla f-\mathbf{v}\|^{2}
$$

where

$\mathbf{v}(x, y)=\left\{\begin{array}{cl}\nabla F(x, y) & \text { if }(x, y) \in \text { retrieved part } \\ \nabla B(x, y) & \text { alternatively } \\ \frac{1}{2}(\nabla F(x, y)+\nabla B(x, y)) & \text { at the boundary }\end{array}\right.$

The retrieved part is restored by using the methods of Section II, before it is used for stitching. Therefore, $F$ is the restored retrieved part.

\section{RESULTS}

Figs. 2 and 4 show examples of crack detection and total variation inpainting. For the graphical representation of detected cracks $M$ we compute their outline using a simple morphological algorithm:

$$
\partial M=(M \oplus B)-(M \ominus B)
$$

where $B$ is the unit disk. The outline is superimposed over the initial painting. In Fig. 5 an example of major damage is 


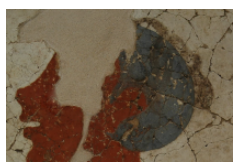

(a) The area to be restored

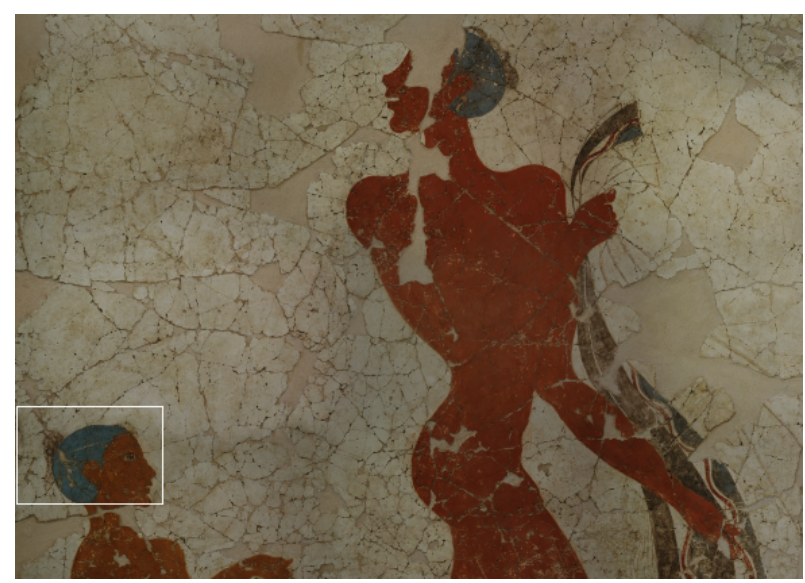

(c) Template matching: the retrieved part is enclosed by the frame

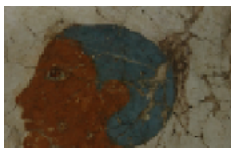

(d) Foreground

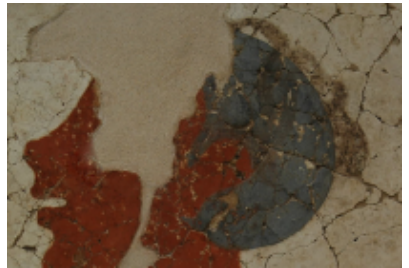

(f) Background

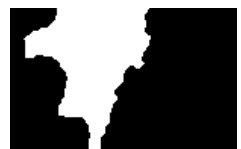

(e) Mask

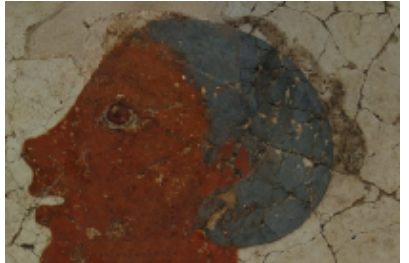

(g) Stitching result
Fig. 7. Example of non-local inpainting in the wall painting Men in Procession. Template matching is used to detect relevant information somewhere else in the wall painting and then image stitching is applied as a final step of the robust inpainting scheme for the large damage restoration.

shown, where GrabCut provides the segmentation mask.

In Figs. 7 and 8 two complete examples of mural areas restoration are presented. Fig. 7 illustrates the restoration of the older man's head in wall painting Men in Procession. The head of the younger man is retrieved automatically, as the template matching algorithm detects it when the template is horizontally flipped. The template is the neighborhood (complement) of the recognized-as-missing area in Fig. 5d. Then the seamless image stitching mechanism based on the algorithm of Tao et al. is applied. The area to be restored and the retrieved part being horizontally reflected are the background and the foreground images, respectively.

In Fig. 8 the restoration of missing information in part

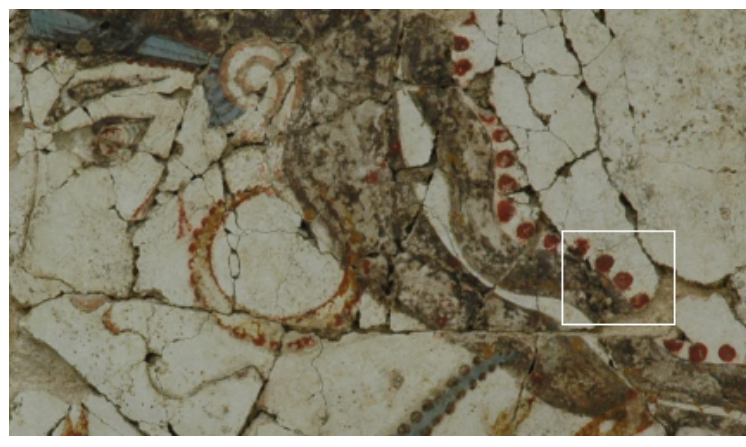

(a) Template matching

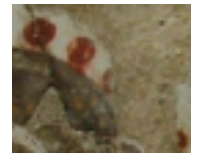

(b) The selected area to be restored
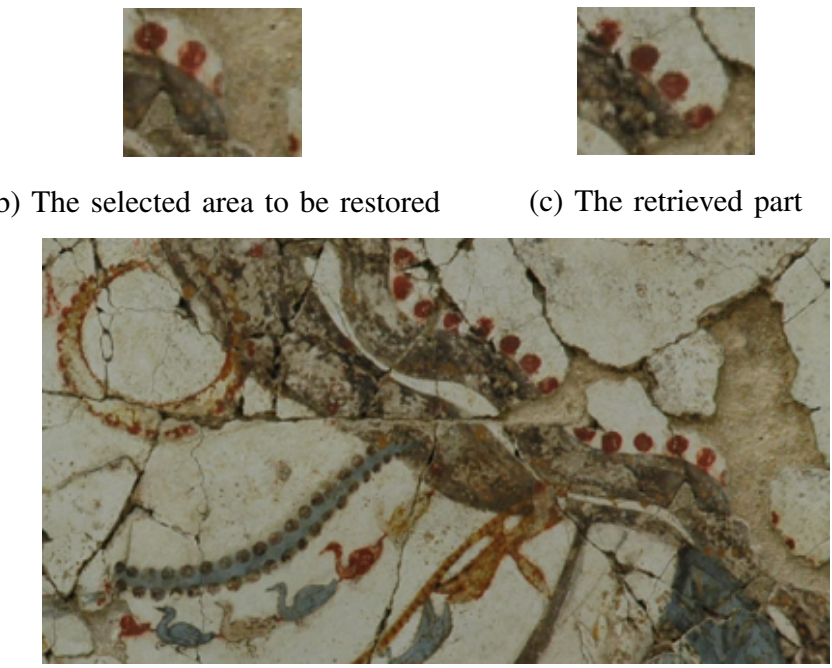

(d) Damaged wall painting

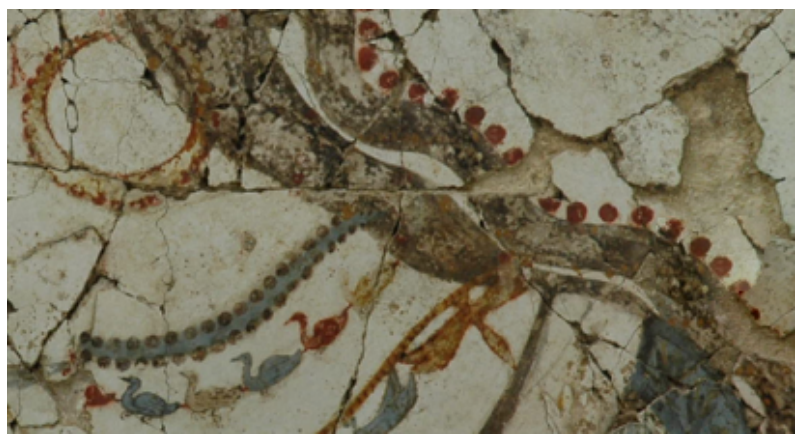

(e) After the partial crown restoration

Fig. 8. Example of non-local inpainting in the wall painting Saffron Gatherer \& Potnia. There is missing information in part of Potnia's crown that is restored by retrieving semantically and geometrically similar part from another area of the crown via template matching.

of Potnia's crown in the wall painting Saffron Gatherer \& Potnia is depicted. It is restored by retrieving semantically and geometrically similar part from another area of the crown via template matching. The template detects the relevant part after a small rotation clockwise. 


\section{CONCLuSion}

We have designed and implemented an integrated system to achieve the digital restoration of Theran wall paintings. As depicted in Fig. 6, by leveraging morphological feature detection, graph cuts, inpainting, template matching and image stitching methods, and by suggesting appropriate algorithms for crack detection, we can satisfactorily restore missing wall painting areas of various sizes and shapes in an automatic way. Integrating local and non-local information yields interesting interpretations of the missing areas, while avoiding the overhead of machine learning.

\section{ACKNOWLEDGEMENT}

We wish to thank George Papandreou, who provided us his high-quality wall painting photographs. During his Ph.D. dissertation [17], he acquired hundreds of photographs of murals preserved at the Akrotiri Thera excavation site and used them to create ultra-high resolution mosaics of each composition. The help of Prof. Christos Doumas, Prof. Andreas Vlachopoulos and Dr. Fragoula Georma at the Center for the Study of Prehistoric Thera is gratefully acknowledged for making the wall paintings available for digitization and their long-term collaboration with the NTUA team. We also thank Eftychios Pnevmatikakis, who generously shared his total variation inpainting source code [21] with us, which was the starting point for implementing the corresponding part in our system.

\section{REFERENCES}

[1] M. Bertalmío, G. Sapiro, V. Caselles and C. Ballester. "Image Inpainting", SIGGRAPH, pp. 417-424, 2000.

[2] M. Bertalmío, L. A. Vese, G. Sapiro and S. Osher. "Simultaneous structure and texture image inpainting", IEEE Transactions on Image Processing, pp. 882-889, 2003.

[3] Y. Y. Boykov and M. P. Jolly. "Interactive graph cuts for optimal boundary \& region segmentation of objects in N-D images", ICCV, vol. 1 , pp. 105-112, 2001.

[4] B. J. Brown, C. Toler-Franklin, D. Nehab, M. Burns, D. Dobkin, A Vlachopoulos, C. Doumas, S. Rusinkiewicz and T. Weyrich. "A System for High-Volume Acquisition and Matching of Fresco Fragments: Reassembling Theran Wall Paintings", ACM Transactions on Graphics, vol. 27 , no. 3,2008 .

[5] J. Canny. "A Computational Approach to Edge Detection", IEEE Transactions on Pattern Analysis and Machine Intelligence, pp. 679-698, 1986.

[6] T. F. Chan, S. Osher and J. Shen. "The digital TV filter and nonlinear denoising", IEEE Transactions on Image Processing, pp. 231-241, 2001.

[7] A. Criminisi, P. Pérez and K. Toyama. "Region filling and object removal by exemplar-based image inpainting", IEEE Transactions on Image Processing, pp. 1200-1212, 2004.

[8] C. Doumas. "The Wall-Paintings of Thera", Thera Foundation, 1992.
[9] I. Giakoumis, N. Nikolaidis and I. Pitas. "Digital image processing techniques for the detection and removal of cracks in digitized paintings", IEEE Transactions on Image Processing, vol. 15, no. 1, pp. 178-188, 2006

[10] J. Hayes and A. A. Efros. "Scene completion using millions of photographs", ACM SIGGRAPH, no. 4, 2007.

[11] L. Joyeux, O. Buisson, B. Besserer and S. Boukir. "Detection and Removal of Line Scratches in Motion Picture Films", IEEE Int. Conf. on Computer Vision and Pattern Recognition, pp. 548-553, 1999.

[12] A. Kokaram, R. Morris, W. Fitzgerald and P. Rayner. "Detection of missing data in image sequences", IEEE Transactions on Image Processing, vol. 4, no. 11, pp. 1496-1508, 1995.

[13] A. Kokaram, R. Morris, W. Fitzgerald and P. Rayner. "Interpolation of missing data in image sequences", IEEE Transactions on Image Processing, vol. 4, no. 11, pp. 1509-1519, 1995.

[14] A. Levin, A. Zomet, S. Peleg and Y. Weiss. "Seamless Image Stitching in the Gradient Domain", ECCV, pp. 377-389, 2004.

[15] P. Maragos. "Morphological Filtering for Image Enhancement and Feature Detection", The Image and Video Processing Handbook, 2nd ed., edited by A. C. Bovik, Elsevier Acad. Press, pp.135-156, 2005.

[16] S. Masnou and J.-M. Morel. "Level Lines based Disocclusion", ICIP, pp. 259-263, 1998

[17] G. Papandreou. "Image analysis and computer vision: theory and applications in the restoration of ancient wall paintings", $\mathrm{PhD}$ Thesis, National Technical University of Athens, 2009. In Greek.

[18] C. Papaodysseus, M. Exarhos, M. Panagopoulos, P. Rousopoulos, C Triantafillou and T. Panagopoulos. "Image and Pattern Analysis of 1650 B.C. Wall Paintings and Reconstruction”, IEEE Transactions on Systems, Man, and Cybernetics, Part A (TSMC) 38(4):958-965, 2008.

[19] C. Papaodysseus, T. Panagopoulos, M. Exarhos, C. Triantafillou, D. K. Fragoulis and C. Doumas. "Contour-shape based reconstruction of fragmented, 1600 BC wall paintings", IEEE Transactions on Signal Processing, vol. 50, no. 6, pp. 1277-1288, 2002.

[20] P. Pérez, M. Gangnet and A. Blake. "Poisson image editing", ACM Transactions on Graphics, vol. 22, no. 3, pp. 313-318, 2003.

[21] E. A. Pnevmatikakis and P. Maragos. "An inpainting system for automatic large structure-texture restoration with text removal", ICIP, pp. 2616-2619, 2008.

[22] C. Rother, L. Bordeaux, Y. Hamadi and A. Blake. "AutoCollage", ACM Transactions on Graphics, pp. 847-852, 2006.

[23] C. Rother, V. Kolmogorov and A. Blake. "GrabCut: Interactive foreground extraction using iterated graph cuts", ACM Transactions on Graphics, vol. 23, pp. 309-314, 2004.

[24] J. Shen and T. F. Chan. "Mathematical Models for Local Nontexture Inpaintings", SIAM Journal of Applied Mathematics, vol. 62, no. 3, pp. 1019-1043, 2002.

[25] H. Shin, C. Doumas, T. A. Funkhouser, S. Rusinkiewicz, K. Steiglitz, A. Vlachopoulos and T. Weyrich. "Analyzing fracture patterns in Theran wall paintings", VAST, pp. 71-78, 2010.

[26] M. W. Tao, M. K. Johnson and S. Paris. "Error-tolerant image compositing”, ECCV, pp. 31-44, 2010.

[27] Research page: http://cvsp.cs.ntua.gr/research/thera/. 